The bronchiectasis severity index derived from these models was composed of prior hospitalisation (5 points), MRC dyspnoea score (0-3 points), $\mathrm{FEV}_{1}$ (0-3 points), bacterial colonisation (0- 3 points) Age ( $0-6$ points) BMI $<18.5$ ( 2 points) Exacerbation frequency (0-2 points) and radiological extent (1 point). The AUC for mortality was $0.80(0.74-0.86)$ and the AUC for hospitalisation was $0.88(0.84-0.91)$. There was a clear difference in exacerbation frequency and quality of life using the St. Georges Respiratory Questionnaire between patients classified as low, intermediate and high risk by the score $(\mathrm{p}<0.0001$ for all comparisons).

In the validation cohorts, the AUC for mortality ranged from 0.81-0.84 and for hospitalisation was AUC 0.80-0.88.

Conclusions The bronchiectasis severity index identifies patients at risk of future mortality, hospital admissions and exacerbations.

\section{S125 A RETROSPECTIVE STUDY CHARACTERISING CILIARY ULTRASTRUCTURE, LIGHT MICROSCOPY AND SPUTUM MICROBIOLOGY ASSOCIATIONS WITH LUNG FUNCTION DECLINE IN A LARGE ADULT PRIMARY CILIARY DYSKINESIA COHORT}

A Shah, A Rogers, A Shoemark, D Bilton, R Wilson, MR Loebinger; Royal Brompton \& Harefield NHS Foundation Trust, London, UK;

10.1136/thoraxjnl-2013-204457.132

Background Primary ciliary dyskinesia (PCD) is an inherited disease related to ciliary dysfunction, with heterogeneity in clinical presentation, prognosis and ciliary ultrastructure. Our study aimed to comprehensively characterise a large cohort with respect to ciliary ultrastructure, beat frequency, sputum microbiology, mortality and lung function decline.

Method A cohort of 100 adult PCD patients was identified at a tertiary respiratory centre. A retrospective analysis of clinical age at presentation and diagnosis alongside ciliary ultrastructure, nasal nitric oxide, beat frequency, sputum microbiology, lung function at diagnosis and follow-up and mortality were recorded. Non-parametric multi-parameter analysis of variance and Spearman rank correlation statistical analysis was performed to identify significant associations with decline in lung function (FEV1\%/year). Median duration of follow-up was 7.5years (range 2-30years).

Results Overall mortality was 4\% (median age of death 55 years). $12 \%$ of patients had a central pair/transposition defect, $37 \%$ missing outer dynein arms, 15\% missing inner dynein arms, $28 \%$ no arms, and 3\% had normal ultrastructure. There was no significant correlation between ciliary ultrastructure, beat frequency (range $0-13.9 \mathrm{~Hz}$ ) and nasal nitric oxide with clinical age at presentation (range 1-26 years) and diagnosis (range 1-72 years) or lung function at presentation and decline with followup. There was additionally no significant association between sputum isolation including Pseudomonas aeruginosa with lung function decline. $44 \%$ of patients had Pseudomonas aeruginosa chronic infection. The incidence of NTM colonisation was low (4\%). Aspergillus species colonisation was additionally low (5\%). The average lung function decline in the cohort was $1.45 \%$ FEV1/year.

Conclusions Comprehensive characterisation of an adult PCD cohort with ciliary ultrastructure, light microscopy, clinical presentation and follow-up data shows a relatively favourable outcome with optimum care. Ciliary ultrastructure, beat frequency and nasal nitric oxide does not predict prognosis. Contrary to parallel diagnoses such as cystic fibrosis and adult idiopathic bronchiectasis, microbiological isolation of Pseudomonas aeruginosa is not associated with a more rapid decline in lung function with optimal prophylaxis and care. Contrary to recent suggestion of low ciliary beat frequency and low nasal nitric oxide association with NTM susceptibility, we did not find a high incidence of NTM or Aspergillus species within this cohort.

\section{S126 MOLECULAR EPIDEMIOLOGICAL ANALYSIS SUGGESTS CROSS INFECTION WITH PSEUDOMONAS AERUGINOSA IS RARE IN NON-CYSTIC FIBROSIS BRONCHIECTASIS}

${ }^{1} \mathrm{~A}$ De Soyza, ${ }^{2} \mathrm{~A}$ Perry, ${ }^{3} \mathrm{AJ}$ Hall, ${ }^{4} \mathrm{~S}$ Sunny, ${ }^{2} \mathrm{KE}$ Walton, ${ }^{5} \mathrm{~N}$ Mustafa, ${ }^{5} \mathrm{~J}$ Turton, ${ }^{5} \mathrm{DT}$ Kenna, ${ }^{3} \mathrm{C}$ Winstanley; ${ }^{1}$ ICM Newcastle University \& Freeman Hospital Bronchiectasis service, Newcastle, UK; ${ }^{2}$ Department of Medical Microbiology, Newcastle upon Tyne Hospitals Trust, Newcastle, UK: ${ }^{3}$ Institute of Infection and Global Health, University of Liverpool, Liverpool, UK; ${ }^{4}$ Sir William Leech Centre for Respiratory Research and Freeman Hospital Adult Bronchiectasis Unit, Newcastle upon Tyne Hospitals Trust, Newcastle, UK; ${ }^{5}$ Public Health England, Colindale, London, UK

\subsection{6/thoraxjnl-2013-204457.133}

Background Non Cystic Fibrosis Bronchiectasis (NCFBr) is a cause of significant morbidity and mortality. Pseudomonas aeruginosa, a key pathogen in $\mathrm{NCFBr}$, is associated with premature mortality. Globally, common clones of P. aeruginosa have been recognised from clinical and environmental sources and in Cystic Fibrosis (CF) cross infection is known to occur. There are no robust data on cross infection in NCFBr. This evidence gap impacts on managing patients but was omitted from the BTS 2010 guidelines due to the paucity of data.

Aims To seek evidence of cross infection amongst $\mathrm{NCFBr}$ patients.

Methods Single centre cross sectional study: We studied $50 \mathrm{P}$. aeruginosa isolates from $40 \mathrm{NCFBr}$ patients using two genotyping techniques (both blinded); an Array Tube (AT) method and Variable Number Tandem Repeat (VNTR) analysis. We included known CF clonal strains as internal controls. We then compared the data using genotype databases.

Results This is the largest cross infection study to our knowledge. We demonstrated that shared $\mathrm{P}$. aeruginosa NCFBr genotypes were infrequent. Twelve patient isolates did not match any other isolate within the NCFBr collection or the databases. The most common clone, clone C $(10 \%)$, is also known to be abundant in the environment. In ten patients where longitudinal isolates were examined, paired isolates gave matching genotyping data suggesting persistent infection. There was incomplete concordance between the Array-Tube and VNTR methods (88\% agreement).

Conclusion There were no dominant Pseudomonas aeruginosa clones in NCFBr suggesting that the most prevalent mode of infection is sporadic and cross infection is rare. This may reflect the local infection control measures however. Multicentre studies are suggested to further assess the risks.

\section{Mechanisms in carcinogenesis}

\section{S127 CHEMOTHERAPY SENSITISES MALIGNANT PLEURAL MESOTHELIOMA CELLS TO UNDERGO MSC-TRAIL INDUCED APOPTOSIS}

KK Kolluri, E Sage, ZQ Yuan, A Gaingreco, SM Janes; University College London, London, United Kingdom

10.1136/thoraxjnl-2013-204457.134 
Spoken sessions

\begin{tabular}{llllllll}
\multicolumn{6}{l}{ Abstract S127 Table 1. Apoptosis induced by recombinant TRAIL and MSC-TRAIL on combination with Vorinostat } \\
\hline & rTRAIL & Vorinostat & $\begin{array}{l}\text { rTRAIL and } \\
\text { Vorinostat }\end{array}$ & $\begin{array}{l}\text { MSCTRAIL } \\
\text { not activated }\end{array}$ & $\begin{array}{l}\text { MSCTRAIL } \\
\text { Activated }\end{array}$ & $\begin{array}{l}\text { MSCTRAIL not } \\
\text { Activated + Vorinostat }\end{array}$ & $\begin{array}{l}\text { MSCTRAIL } \\
\text { Activated + Vorinostat }\end{array}$ \\
\hline Ju77 & $7.71 \%$ & $51.35 \%$ & $80.77 \%$ & $10.32 \%$ & $48.73 \%$ & $47.44 \% \%$ & $77.7 \%$ \\
CRL2081 & $56.75 \%$ & $78.95 \%$ & $96.6 \%$ & $37.3 \%$ & $57.63 \%$ & $81.45 \%$ & $90.93 \%$ \\
One58 & $13.41 \%$ & $43.97 \%$ & $79.27 \%$ & $10.88 \%$ & $53.8 \%$ & $49.25 \%$ & $77.8 \%$ \\
\hline
\end{tabular}

MPM cells are treated with recombinant Trail $(100 \mathrm{ng} / \mathrm{ml})$ and Vorinostat $(2.5 \mu \mathrm{M})$. MSC are plated in 1:1 ratio with tumour cells. MSC are activated with doxycycline to induce TRAlL expression.

Introduction Malignant pleural mesothelioma (MPM) is a highly aggressive, incurable, chemoresistant tumour. Recent studies have shown that Mesenchymal stem cells (MSC) can home to and incorporate into the tumour stroma. Their tumour tropism can be used to deliver Tumour necrosis factor related apoptosis inducing ligand (TRAIL), a transmembrane protein that selectively induces apoptosis in transformed cells. However, not all tumours are sensitive to TRAIL. TRAIL works through triggering the extrinsic apoptotic pathway while conventional chemotherapeutic agents act by triggering the intrinsic apoptotic pathway. We hypothesised the crosstalk between these two pathways could be exploited by combining chemotherapy and MSC-TRAIL in MPM tumour cell lines.

Methods MSC were engineered to express TRAIL using a lentiviral plasmid vector. A Tetracycline (Tet)-inducible system was used as a backbone to control the expression of TRAIL. Apoptosis induced by recombinant TRAIL, MSC-TRAIL in MPM cell lines on combination with Vorinostat, a chemotherapeutic agent, was measured by Annexin-V/DAPI based flow cytometry.

Results The combination of recombinant TRAIL and Vorinostat act synergistically to induce apoptosis in MPM cell lines. Recombinant TRAIL and Vorinostat, as monotherapies induce $7.17 \%$ and $51.35 \%$ apoptosis in an MPM cell line JU77 respectively. In CRL2081 and ONE58 cell lines, recombinant TRAIL induces $56.75 \%$ and $13.41 \%$ apoptosis while Vorinostat leads to $78.95 \%$ and $43.97 \%$ apoptosis respectively. The combination of recombinant TRAIL and Vorinostat shows an increased amount of apoptosis in JU77, CRL2081 and ONE58 cell lines at $80.77 \%, 96.6 \%$ and $77.27 \%$ respectively (Table 1 ).

Similar synergistic affect was observed when TRAIL expressing MSCs were co-cultured with Vorinostat treated MPM cell lines. MSC-TRAIL induced apoptosis in JU77 (48.73\%), CRL2081 (57.63\%) and ONE58(53.8\%). Combined treatment of Vorinostat and MSC-TRAIL significantly increased apoptosis to $77.7 \%$ in JU77, $90.93 \%$ in CRL2081 and $77.8 \%$ in ONE5 8 cells (Table 1).

Conclusion The combination of Vorinostat and recombinant TRAIL acts synergistically to induce apoptosis in malignant plural mesothelioma cells. Similar affect is observed with the combination of MSC-TRAIL and Vorinostat. This study indicates that Mesenchymal stem cells can be used as vectors for delivery of TRAIL and upon combination with Vorinostat, could be a potential therapy for malignant pleural mesothelioma.

\section{S128 REDUCTION OF LUNG METASTASIS BY ENGINEERED MESENCHYMAL STEM CELLS EXPRESSING SECRETED SOLUBLE TRAIL}

ZQY Yuan, KK Kolluri; University College London, London, UK

\subsection{6/thoraxjnl-2013-204457.135}

Bone marrow-derived mesenchymal stem cells (MSC) are promising tools for cancer therapy because they are able to home to and incorporate within tumour tissue. Tumour necrosis factor-related apoptosis-inducing ligand (TRAIL) is a pro-apoptotic protein that induces selective apoptosis of tumour cells, while sparing normal cells. Therefore it is expected that MSCs engineered to produce TRAIL would home to and kill cancer cells in a lung metastatic cancer model. Two lentiviral vectors were constructed to express the full-length (FL) TRAIL and a truncated soluble form (ILZ-sT), respectively. A secretion peptide and an isoleucine zipper (ILZ) peptide were added to the N-terminal of the soluble form to force its secreted expression and to enhance its trimerization. Human MSCs were transduced with viruses and both constructs produced soluble TRAIL into cell media that can rapidly induced apoptosis of cancer cells. However the ILZ-sT fusion construct expresses significantly higher level of soluble TRAIL, and causes better in vitro lung cancer cell (A549) killing than the FL one.

In coculture experiments both construct viruses transduced MSCs caused lung (A549), breast (MDAMB231), squamous (H357), and cervical (Hela) cancer cell apoptosis and death with similar efficiencies. A synergistic effect of cancer cell killing was observed for the combinational treatment of MSC-TRAIL cells with Saha, a histone deactylase inhibitor. When systemically delivered both MSC-FLT and MSC-ILZ-sT cells showed significant reduction of lung metastasis in a pulmonary metastasis murine model. Interestingly, ILZ-sT expressing cells demonstrated higher efficiency of metastasis reduction than FLT cells. These findings suggests that TRAIL expressing MSCs particularly ILZsT cells could be potentially developed as a therapy for lung metastasis diseases.

\section{S129 THE NATURAL HISTORY OF BRONCHIAL PRE-INVASIVE DISEASE}

JM Brown, G Hardavella, B Carroll, M Falzon, N Navani, PJ George, SM Janes; University College London Hospital, London, UK

\subsection{6/thoraxinl-2013-204457.136}

Background Bronchial pre-invasive lesions represent the earliest stages of the stepwise progression of squamous carcinogenesis, they predominantly affect the large airways and are readily detectable using autofluoresence bronchoscopy (AFB) however very little is known about the natural history of these lesions and no randomised data exists to determine whether intervention before progression to invasion improves outcome.

Methods A total of 94 patients with bronchial dysplasia were enrolled into an on-going surveillance cohort at University College London Hospital running prospectively since 1999. Lesions were biopsied longitudinally and kept under regular surveillance with AFB and low dose annual CT scanning until resolution or progression to invasive disease occurred. Retrospective analysis of lesional destiny was undertaken to determine the proportions of progressive vs. regressive lesions that occur in low grade dysplasia (LGD- squamous metaplasia, mild and moderate dysplasia) 